\title{
Alter
}

Revue de phénoménologie

$21 \mid 2013$

La Vie

\section{Le phénomène de la vie au prisme des pensées de Merleau-Ponty et de Jonas}

\section{Eric Pommier}

\section{(2) OpenEdition}

\section{Journals}

Édition électronique

URL : http://journals.openedition.org/alter/842

DOI : 10.4000/alter.842

ISSN : 2558-7927

Éditeur :

Association ALTER, Archives Husserl (CNRS-UMR 8547)

\section{Édition imprimée}

Date de publication : 1 novembre 2013

Pagination : 171-186

ISBN : 978-2-95-223749-9

ISSN : $1249-8947$

\section{Référence électronique}

Eric Pommier, «Le phénomène de la vie au prisme des pensées de Merleau-Ponty et de Jonas », Alter [En ligne], 21 | 2013, mis en ligne le 01 juin 2019, consulté le 07 juillet 2019. URL : http:// journals.openedition.org/alter/842 ; DOI : 10.4000/alter.842 


\section{LE PHÉNOMÈNE DE LA VIE AU PRISME DES PENSÉES DE MERLEAU-PONTY ET DE JONAS.}

Eric Pommier

Le projet de Hans Jonas, et son engagement de philosophe, résulte de la prise de conscience de "l'oubli », dans l'histoire de la philosophie, de la vie. "L'ontologie de la $\operatorname{mort}^{1} »$, qui commande de manière sous-terraine cette histoire, mène à l'échec toute tentative pour la penser, en ramenant l'ambiguïté dont elle est le lieu, soit à la figure univoque de l'organisme étudiée par le biologiste, soit à celle du vécu ou de l'idée, exploré par le phénoménologue ou l'idéaliste. Le dualisme, qui est à l'arrière-plan de ces essais, mène toujours dans une impasse l'effort pour penser l'être et pour accéder à la vérité. Faute de penser la vie, c'est en effet aussi bien notre prétention à comprendre l'unité de l'être qui avorte - puisque le phénomène de la vie déjoue le partage de l'identité matérielle et de la réflexion consciente - que notre aspiration à la vérité, puisque nous ne savons pas comment un vécu subjectif peut s'accorder à une " réalité extérieure " ( $s^{\prime}$ il y en a une) ou comment une réalité extérieure peut s'intérioriser, se dédoubler sous la forme d'une représentation psychique censée en rendre compte. Décrire le mode d'accès au phénomène de la vie, qui déjoue la partition du sujet et de l'objet, permettrait alors de reformuler le sens que nous donnons au terme de vérité, dès lors qu'elle ne consiste plus en une saisie représentative d'un objet ou en la "production » d'une telle représentation. Il convient cependant de mettre à l'épreuve cette ambition même de Jonas, afin de s'assurer que sa démarche ne demeure pas encore trop dépendante de l'ontologie de la mort et d'un dualisme de l'ombre. Et c'est ce que nous souhaitons

${ }^{1}$ Hans Jonas, Le Phénomène de la vie, trad. D. Lories, Bruxelles, De Boeck, 2000. Désormais noté PV. 
réaliser par le moyen d'un rapprochement avec l'œuvre de MerleauPonty. Il s'agira, en premier lieu, de rappeler en quel sens, chez l'un et l'autre auteurs, l'ouverture au monde exige de prendre en considération la vie corporelle. Dans un second temps, il faudra mettre en lumière la manière dont chacun des deux auteurs peut mettre au jour les insuffisances de l'autre et ainsi faire allusion à la ligne de fuite permettant d'assigner un horizon à la pensée de la vie.

La tâche que s'assigne Jonas consiste à reprendre à nouveaux frais le problème de l'être tel que la tradition philosophique nous l'a légué. Cela signifie, en une proximité avec le vœu heideggérien de destruction, qu'il faut prendre en compte les conditions actuelles dans lesquelles ce problème se pose, sans pour autant rester dépendant de cette situation. La question ontologique ne doit donc pas s'affranchir des conditions dans lesquelles l'histoire de la pensée nous l'a transmise, comme si la question devait se poser indépendamment de toute histoire, mais elle ne doit pas non plus rester enclose dans les paramètres ontologiques propres au moment présent. L'héritage doit donc être assumé en vue d'une réappropriation authentique qui permet de libérer le sens de la question et d'y apporter une réponse libérée des apories auxquelles cette question a été confrontée du fait même de sa formulation traditionnelle. C'est ainsi que Jonas entreprend une interprétation de l'histoire des interprétations de l'être et y décèle un singulier oubli de la vie. Le dualisme de la période classique brise l'unité moniste du panvitalisme des origines et prépare l'avènement de deux nouveaux monismes, matérialiste et «idéaliste ». C'est en effet en conséquence de la distinction pure entre la res extensa et la res cogitans qu'on peut ensuite résoudre le problème de l'unité de l'être légué par le dualisme cartésien en réduisant le tout du réel à une des deux substances pour en faire dériver l'autre.

Nous pouvons faire trois remarques relatives à cette lecture, ici survolée, de l'histoire de la tradition. En premier lieu, le dualisme classique n'est qu'une résurgence, mais conceptuellement plus précise et rigoureuse, de la crise de la période gnostique qui a conduit à briser l'unité cosmique léguée par les Grecs. En second lieu, ce dualisme conserve une actualité et une influence, y compris au sein de la pensée contemporaine. Il est à l'arrière-plan de ses idéalismes et de ses matérialismes. Ainsi par exemple, aux yeux de Jonas, la pensée de Heidegger n'est qu'une réactualisation du gnosticisme et du dualisme qui en est la colonne vertébrale. En troisième lieu, c'est précisément ce dualisme de l'ombre qui condamne à l'échec les prétentions ontologiques de ces pensées. De fait, il s'agit toujours pour elles d'en venir à retrouver celle des deux substances qu'elles n'ont pas 
prise pour norme ontologique, ce qui donne lieu à des apories indépassables. Le matérialisme doit rendre compte de l'«immatérialité » de l'esprit; l'idéalisme doit rendre compte de la réalité étendue et résistante de la matière. En outre, chacun des deux doit justifier sa capacité à tenir un discours vrai, à savoir comment une représentation peut s'accorder à une réalité « extérieure ». C'est ces impasses qui invitent à dépasser les conditions mêmes du problème tout en en tenant compte. Il faut donc rejeter le dualisme tout en conservant le souci de penser la dualité, mais depuis une base ontologique qui en éclaire la possibilité.

Or c'est manifestement à condition de rejeter les catégories métaphysiques de l'âme et du corps appréhendées de manière substantielle qu'il nous sera possible de penser la vie pour elle-même, et partant de dénouer le nœud à partir duquel la question de l'être doit être abordée. C'est en interrogeant «la vie», sur fond de laquelle l'esprit semble se détacher, et à partir de laquelle nous pouvons nous ménager un accès authentique à l'être de la matière, que nous serons à mêmes de délivrer le sens d'être de la dualité sans le prédéterminer. C'est donc en mettant en œuvre une approche phénoménologique plutôt que métaphysique que nous pourrons accéder au sens d'être du phénomène de la vie.

De ce point de vue, il faut relever un incontestable air de famille entre la démarche que Merleau-Ponty met en œuvre dans La Structure $d u$ comportement ${ }^{2}$ et celle de Jonas au sein du Phénomène de la vie. L'analogie d'approche est éclairante et permet de progresser dans notre souhait de circonscrire l'espace d'une phénoménologie de la vie. Trois remarques permettront de justifier un tel rapprochement. C'est d'abord au plan même de la finalité du projet que les deux auteurs se rencontrent. Ainsi Merleau-Ponty considère que c'est à condition de partir du phénomène du comportement «neutre à l'égard des distinctions classiques du "psychique" et du "physiologique $^{3 \prime \prime}$ ", que non seulement nous pourrons comprendre les rapports de la conscience avec la nature mais en outre que nous pourrons redéfinir le sens même de l'âme et du corps. C'est donc à condition de rompre d'emblée avec le dualisme que nous pourrons comprendre le sens véritable de la dualité et affronter le questionnement ontologique $^{4}$. Et si $\mathrm{c}^{\prime}$ est la notion de comportement vivant qui vient au

\footnotetext{
2 Maurice Merleau-Ponty, La structure du comportement, Paris, Puf, «Quadrige», 2002. Désormais noté SC.

${ }^{3}$ SC, p. 2.

${ }^{4}$ De même, c'est à condition de rejeter l'opposition de l'objectivisme et de l'analyse transcendantale purement critique que la question de la vérité doit être abordée.
} 
premier plan, ce n'est pas seulement que, contrairement à l'explication physique du comportement de la matière ou à l'explication psychologique qui fait de la conscience tantôt une chose matérielle tantôt une chose spirituelle, l'organisme vivant est en lui-même le lieu d'une ambiguïté fondamentale, comme en témoigne le débat entre le mécanisme et le vitalisme, propice à une mise en question immédiate de son mode d'être, c'est aussi et surtout parce que c'est l'homme dans sa prétention à agir et à comprendre qui est au principe de la démarche philosophique. Or le comportement humain ne peut être compris indépendamment de son activité nerveuse grâce à laquelle il se rapporte à son environnement. Ainsi la notion de comportement véhiculée par le behaviorisme a donc le grand mérite de déspiritualiser l'homme. Néanmoins, comme le fait remarquer Merleau-Ponty cette déspiritualisation ne doit pas pour autant conduire à un mouvement inverse de rematérialisation. Le comportement doit rester neutre vis-à-vis de cette partition ontologique de la matière et de l'esprit afin de comprendre l'homme comme "débat et "explication" perpétuelle avec un monde physique et avec un monde social $»^{5}$.

Or, de manière symétrique, Hans Jonas lui aussi, entend proposer une interprétation existentielle des faits biologiques qui échappe à l'opposition du sujet et de l'objet. Parce que l'esprit n'est jamais à ce point immatériel qu'il ne faille lui reconnaître un enracinement organique et parce que la matière n'est jamais à ce point close sur ellemême qu'elle ne manifeste en son sein "un esprit d'ouverture », ce dont témoigne le phénomène de l'évolution, il faut comprendre la vie de manière neutre au regard du découpage a priori d'un esprit purement intérieur et d'une matière purement étendue. En outre, ici encore le témoignage de l'homme à propos de son comportement vivant devra être privilégié, dans la mesure même où ce comportement récapitule, pour ainsi dire, les différentes régions ontologiques de la matière, de l'organique et de l'esprit ${ }^{6}$. C'est d'ailleurs en vertu de son appartenance ontologique au royaume de la vie que l'homme pourra reconnaître les autres vivants au sein de son environnement ${ }^{7}$.

Par-delà même la prétention commune à rendre compte du phénomène du comportement vivant en tant que clef de voûte de l'investigation ontologique, les deux philosophes se rejoignent, c'est le second point de convergence, sur le contenu même de la description du phénomène de la vie. Effectivement, rejetant le mécanisme de la

\footnotetext{
${ }^{5}$ SC, p. 3 (note 2).

${ }^{6}$ PV, p. 33.

7 PV, p. 92.
} 
science et la réflexion de la conscience, Merleau-Ponty comprend le comportement à partir des notions de structure et de forme. La forme s'applique aussi bien au champ physique, biologique que psychologique mais selon trois structures différentes. Au lieu d'opposer la matière et sa quantité, la vie et son ordre, l'esprit et sa valeur, voici désormais une même forme dont la structure varie en mettant au premier plan tel ou tel de ces caractères selon l'ordre phénoménal considéré. La différence structurale doit se comprendre en termes de degré d'intégration de la forme de manière que l'individualité $s^{\prime} y$ réalise toujours davantage selon que l'on envisage la structure physique, biologique ou psychologique. Une telle description a le double avantage de rejeter aussi bien le réductionnisme, par exemple matérialiste - puisqu' on ne pourra pas dire que la forme organique ou psychologique est de même nature que la forme physique - que le séparatisme ontologique, qui lézarde la nature, en affirmant la différence radicale de ces structures entre elles.

La structure biologique occupe une place particulière dans l'approche merleau-pontyenne, puisqu'elle permet de mettre au jour la dimension dialectique propre au vivant. Alors que la forme physique résulte d'un équilibre à l'égard des conditions extérieures, alors que la réaction du système matériel a pour but la réduction d'une tension, l'acheminement vers un état de repos, voici que la forme vivante obtient son équilibre non par rapport à des conditions présentes mais par rapport à des conditions possibles que le système a précisément pour vocation d'amener à l'existence. Autant dire que l'organisme ne se contente pas de s'adapter à des conditions données de manière mécanique mais qu'il s'y ajuste en préférant certains comportements à d'autres. Le vivant n'agit pas en conséquence d'une perception qui s'impose à lui ; il perçoit de manière à agir selon des possibilités privilégiées. L'excitant n'est pas passivement reçu mais activement élaboré pour rendre possible l'action préférée. Sans être créateur de son milieu, l'animal n'est pas pour autant déterminé par lui ou moulé sur lui, puisqu'il s'y rapporte sur le mode d'une réponse possible à une question posée. Et la signification vitale excède toujours les propriétés matérielles du vivant et de son environnement. Avec la vie, l'être s'ouvre à l'expression d'une signification qui traduit l'orientation d'un possible tourné vers l'extériorité qu'il contribue à éclairer. C'est une telle dialectique qui connaît un développement supplémentaire dans le cas de l'homme. C'est en effet la catégorie du travail qui permet de comprendre comment l'homme transforme la nature conformément au sens du possible, sans que la différence anthropologique d'avec l'animal ne doive se comprendre 
en termes substantiels, l'âme se juxtaposant à l'activité instinctive du corps. De fait l'avènement de l'ordre supérieur ne laisse pas indemne l'ordre inférieur mais au contraire l'investit d'un sens nouveau. On ne séparera donc pas le psychisme du somatique et comme l'écrit Merleau-Ponty, «l'homme ne peut jamais être un animal $»^{8}$.

Il suffisait de faire ce bref rappel pour mettre en valeur la communauté de vue frappante avec la démarche de Jonas. L'approche substantialiste, ici encore, doit être écartée si l'on veut comprendre les différences ontologiques régionales au sein d'une approche unitaire de l'être. Matière, vie, esprit ne sont pas des unités ontologiques autonomes qui démembrent l'unité de la nature mais des ordres de signification au sein d'une seule et même conception de l'être dont les différences témoignent d'un progrès toujours plus grand de l'individualité face à un monde toujours plus ouvert. Ainsi de l'animal à l'homme, c'est une même vie qui s'affirme à ceci près que le soi $\mathrm{s}^{\prime}$ affirme davantage et que les horizons se révèlent plus amples. Au premier, il faut reconnaître la largeur de vue et d'action que permettent la motricité, la perception et l'émotion - grâce à eux des horizons spatiaux et temporels s'ouvrent; c'est ce qui faisait défaut à la plante. Au second, il faut reconnaître le pouvoir d'imaginer et de symboliser, grâce auquel la liberté du soi s'accroît d'autant, notamment jusqu'à pouvoir faire retour sur lui-même. Ce n'est donc pas seulement le contrôle moteur que rend possible l'imagination et qui se traduit par le développement des techniques qui lui est offert, c'est aussi le pouvoir de se représenter le soi. Ce progrès de la vie manifeste la transcendance d'un soi ouvert au monde, le surgissement de possibilités, que Merleau-Ponty avait déjà su mettre au jour.

Ici encore le qualificatif de dialectique convient puisque la liberté du vivant s'affirme dans un débat toujours plus vif avec l'environnement au fur et à mesure des progrès de la vie. L'animal qui ouvre un monde de manière relativement individuelle continue cependant de $\mathrm{s}^{\prime} \mathrm{y}$ insérer, tandis que l'homme a le pouvoir de reconfigurer la nature, de la transformer pour y affirmer ses possibilités les plus propres. Avec Jonas, peut-être davantage qu'avec Merleau-Ponty, cette exigence de liberté s'affirme avec nécessité, car si l'espèce ne transforme pas son milieu, si elle ne met pas en œuvre sa liberté alors c'est sa vie, en tant qu'elle est cette espèce, qui sera en péril.

Quoi qu'il en soit, chez Jonas encore, c'est la notion de forme qui permet d'unifier les différents ordres de phénomène. Il existe une forme physique comme il existe une forme vivante bien qu'il faille

8 SC, p. 196. 
distinguer les deux, car si la première résulte de ses composants matériels, en revanche la seconde rend compte de leur renouvellement. La souveraineté ou l'indépendance de cette forme à l'égard de la matière est telle qu'elle exige le remplacement des entités matérielles et que c'est en ce remplacement que consiste la vie, puisque leur conservation statique signifierait la mort de l'organisme, son retour à la matérialité. On comprend que pour trouver une matière à sa disposition cette forme doit s'ouvrir au monde étranger tout autant qu'à elle-même, afin de discriminer dans cet environnement ce qui lui agrée et ce qui lui répugne. De ce point de vue, c'est la notion de métabolisme, véritable analogue jonassien du comportement chez Merleau-Ponty, qui permet de comprendre le sens de la vie. Le vivant demeure en effet le même formellement sous la condition d'un renouvellement matériel constant qui suppose un échange avec les alentours. Chez Jonas comme chez Merleau-Ponty, la transcendance de la vie permet d'ouvrir un monde, de faire surgir des horizons permettant à une individualité de s'affirmer. Chez l'un et chez l'autre, la vie n'est pas une énigme présente au cœur de l'être mais contribue, au contraire, à en éclairer le sens, nervuré de différences qui ne sont pas exclusives de son unité.

Nous pouvons alors mettre l'accent sur le dernier point de convergence, auquel nous avons déjà en réalité fait allusion et qui se focalise sur la méthode employée par les deux auteurs. Aussi bien chez le philosophe français que chez le philosophe allemand, c'est à partir du point de vue du sujet incarné que le phénomène de la vie peut être interprété comme vie. Ainsi Jonas, comme nous l'avons déjà suggéré, revendique la valeur méthodologique de l'anthropomorphisme. Pour peu qu'on rejette la partition dualiste de l'être, le point de vue de l'homme sur la vie ne peut plus être ignoré sous prétexte qu'il serait une projection subjective opérée sur une réalité objective. Une telle accusation suppose en effet l'opposition du sujet et de l'objet que la forme vivante dément. C'est au contraire parce que l'homme se sait vivant qu'il peut reconnaître au dehors d'autres vivants et une telle interpolation de l'acte d'auto-renouvèlement de soi, caractéristique de l'activité métabolique, ne signifie pas ipso facto le transfert illégitime de la substance pensante sur une substance étendue qui n'aurait rien à voir avec elle. Simplement, j'identifie dans mon monde d'autres vivants eux-aussi ouverts au monde ${ }^{9}$. C'est la raison pour laquelle Jonas accorde une importance de premier ordre au témoignage corporel. Mon corps se présente en effet comme l'articulation

9 PV, p. 92. 
de l'intensif et de l'extensif, comme l'unité de l'intérieur et de l'extérieur, ou comme "auto-transcendance même dans chacune des deux directions $»^{10}$. Il n'y a d'âme comme âme qu'en tant qu'elle se relie à un corps; il n'y a de corps comme corps vivant qu'en tant qu'il se relie à l'âme. C'est depuis mon corps que peut s'attester le sens de la vie en général, une ouverture primordiale au monde.

De prime abord, il n'en va guère différemment chez MerleauPonty. Les deux premiers chapitres de La Structure du comportement ont certes pour finalité de procéder à une critique interne de l'approche behavioriste du vivant en montrant qu'elle ne peut rendre compte ni des comportements inférieurs ni des comportements supérieurs à partir de ses seuls présupposés ${ }^{11}$. Cette dénonciation du mécanisme scientifique aurait certes pu être opérée par la voie courte de la réflexion mais cette voie longue permet précisément de rejeter le mécanisme tout en entérinant l'insuffisance du point de vue réflexif : le comportement vivant qui n'est pas mécanique ne relève pas pour autant d'une idée, d'où l'importance de la notion de forme qui permet de neutraliser l'opposition de la matière et de l'idée, tout en faisant droit aux différences structurelles entre les formes physique, vivante et psychique. Cette approche indirecte du comportement vivant mise en œuvre dans les premiers chapitres prépare ainsi l'approche plus directe du troisième chapitre, où la forme vivante est décrite pour elle-même et par comparaison avec la forme physique et la forme psychologique.

Néanmoins, même si c'est la critique interne de la science qui est préalablement mise en œuvre, complétée ensuite par une description des différentes structures prises par la forme, il faut reconnaître que celles-ci ne sont possibles que sous la condition de la reconnaissance par le sujet de sa propre incarnation. C'est à partir du sujet, qui se comprend comme étant son corps, que la reconnaissance des autres formes vivantes est possible et que l'organisme perçu est compris comme perception d'un organisme vivant. L'organisme n'est pas perçu comme étant une apparence trompeuse mais comme un phénomène ayant une signification propre, et ceci non en vertu d'un raisonnement par analogie, mais grâce à une interprétation immédiate, puisque c'est directement que l'autre vivant est compris comme vivant et non grâce à une comparaison. À supposer même qu'il faille dénoncer là un anthropomorphisme, encore faudrait-il expliquer

\footnotetext{
${ }^{10}$ PV, p. 29.

${ }^{11}$ Nous n'y insistons pas mais Jonas également procède à une critique de l'explication scientifique du vivant : par exemple, PV, Essai V.
} 
ce qui peut susciter cet anthropomorphisme, à partir de la chose même $^{12}$. De même que Jonas comprend la vie des autres vivants à partir de celle du sujet qui questionne son mouvement et sa perception, à condition que cette vie ait subi une opération de réduction, permettant d'en comprendre la dimension intentionnelle ${ }^{13}$, on peut dire que Merleau-Ponty subordonne la question de la perception de $l^{\prime}$ organisme vivant à celle du statut du sujet percevant, sous condition de le concevoir après la réduction phénoménologique ${ }^{14}$. C'est ce qui apparaît dans le quatrième chapitre de La Structure du comportement. De fait l'opération de critique interne des sciences, opérée dans les chapitres préalables, suppose ultimement cette expérience de la vie, comprise comme perception "vécue» du phénomène du vivant. Ainsi loin que l'ordre humain de la conscience soit un ordre qui s'ajoute ou se juxtapose aux deux autres ordres physique et organique, il en est bien plutôt la condition de possibilité.

Mais cette conscience est une conscience incarnée, comme telle perceptive et non purement intellectuelle. Le corps n'est pour elle ni objet ni idée mais ce grâce à quoi je peux avoir un accès au monde. C'est par son «moyen » que je peux accéder à la profondeur du monde et échapper à l'alternative du subjectivisme relativiste et de l'objectivisme naïf. La chose perçue n'est ni en soi ni pour l'esprit. Elle se donne à travers des esquisses qui en sont la manifestation : que ces dernières soient toujours partielles ne nous condamnent pas au relativisme mais atteste au contraire de la transcendance de la chose. Le perspectivisme est donc une loi de la chose. Il n'y a pas lieu de faire de l'âme, du corps et de la chose trois secteurs bien distincts au sein de la réalité, puisqu'ils dessinent en vérité un champ unique : les intentions de l'âme sont portées par le corps qui nous ouvre au monde dans la perception. C'est alors parce que je me sais ouvert à lui, grâce à mon corps, que j'y reconnais immédiatement les corps pourvus de cette même ouverture ${ }^{15}$. Tout comme chez Jonas, il n'y a pas ici à poser le problème husserlien de l'intersubjectivité transcendantale : le sujet, comme vivant, est d'emblée ouvert au monde dans toute sa profondeur et les autres sujets y logent en creux.

En dépit du rapprochement que l'on peut opérer entre MerleauPonty et Jonas sur leur commune capacité à circonscrire l'espace d'une phénoménologie de la vie, il faut en effet mettre en évidence la

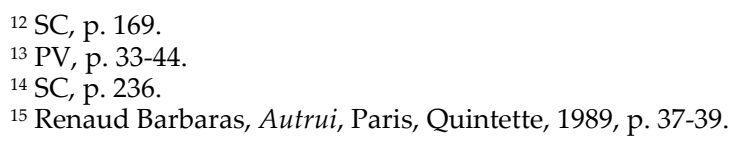


valeur d'une critique réciproque de ces deux approches. Alors, en effet, que l'approche du premier pourrait être qualifiée d'idéaliste par le second, à l'inverse nous pourrions faire valoir que la seconde pourrait être dénoncée comme matérialiste par le premier. Justifions cette double affirmation. De fait, nous pourrions dire que la démarche de Merleau-Ponty souffre de sa dépendance primordiale à la dualité du sujet et de l'objet qui s'accuse sous la forme d'un primat du sujet et qui reconduit la méthode de l'idéalisme, au détriment même de la prétention à penser par-delà le découpage dualiste de l'être. C'est ce qui apparaît dans le dernier chapitre de La Structure du comportement dans lequel Merleau-Ponty, contre toute attente, reconduit une posture transcendantale, référant l'appréhension du comportement à la saisie d'une signification par la conscience. Certes, celle-ci se veut incarnée et non intellectuelle, coupée de ses racines vitales. Certes, le partage de l'âme et du corps ne saurait être « réel » mais relève d'une distinction entre deux ordres de signification. La perception vécue n'est elle-même interprétable ni en termes de représentation psychique ni d'explication causale. Pour autant, tout se passe comme si la réduction reconduisait le présupposé d'une conscience pouvant exister à part du corps et comme si elle imposait son mode d'être aux réalités décrites. Loin que la vie soit comprise à partir d'elle-même au point de donner lieu, ensuite, à la conscience, loin qu'une genèse de la conscience soit effectuée à partir de la vie, $c^{\prime}$ est en vérité la conscience qui s'avère première et qui impose son mode d'être à la vie. Tout se passerait alors comme si au lieu de penser l'émergence de la conscience perceptive à partir de la vie, le philosophe pensait la vie à partir du point de vue d'une conscience perceptive, dont le mode d'être restait déterminé par l'opposition du sujet et de l'objet $^{16}$.

Du point de vue des attendus d'une phénoménologie de la vie, la pensée de Jonas représente donc une avancée au regard de la démarche merleau-pontyenne. En effet, en s'inspirant du couple conceptuel présent chez Whitehead de l'immédiateté de présentation et de l'efficacité causale ${ }^{17}$, Jonas justifie à partir de la vie la tendance inhérente à la perception à objectiver l'être et à se comprendre comme acte d'un sujet contemplatif. Tout en partageant l'ambition merleaupontyenne de rompre avec l'antinomie de l'explication objective et de la définition intellectualiste de la perception, il se donne les moyens

\footnotetext{
${ }^{16}$ Nous ne pouvons développer davantage ce point ici. Cf. Renaud Barbaras, Introduction à une phénoménologie de la vie, Paris, Vrin, 2008, p. 66-73.

17 PV, p. 39.
} 
de dépasser ce dualisme, autrement que par le recours à une opération de réduction qui reste dépendante du point de vue de la conscience, puisqu'il saisit à sa racine, c'est-à-dire dans sa genèse, le principe même en vertu duquel la vie est condamnée à s'illusionner sur elle-même. En d'autres termes, c'est parce que la vie s'ouvre au monde à travers la perception et c'est parce que la perception est originairement vouée à l'objectivation que le vivant percevant est voué à se représenter comme sujet conscient. C'est du fond même de la vie que la dualité émerge et que son sens véritable se masque ultérieurement sous la figure trompeuse de l'interprétation dualiste.

Tel est le rôle décisif, peu souligné, de l'essai VI du Phénomène de la vie consacré non pas d'abord à la phénoménologie de «la » perception mais à la phénoménologie "des » sens. Dans une étude comparée de la manière dont le monde se donne aux sens, Jonas montre en effet que la vue se distingue notamment par son pouvoir de distanciation et de neutralisation « causale ». Alors que la perception en général permet au vivant de déployer un horizon spatial, il faut en effet reconnaître que le primat de la vue tend à «effacer» le monde au profit d'une appréhension objectivante de la nature, tel qu'un sujet détaché du monde peut s'en faire la représentation, du fait même de son absence d'implication en elle, dès lors que la vision tend à effacer le débat qui existe entre la chose et le sujet qui s'engage en elle ou qui est « interrogé » par elle. Une telle idéalisation du monde est certes à l'avantage de l'agir comme de la spéculation, puisqu'en nous donnant une vision déterminée et sans profondeur de ce monde, nous pouvons, d'une part, mieux agir sur les choses, d'autre part, mieux nous les représenter et les expliquer ${ }^{18}$. Mais ce mouvement d'objectivation est également une perte au plan ontologique. C'est donc seulement en procédant à une critique de la dimension idéalisante de la perception visuelle que nous pourrons renouer avec le sens originaire de la perception, en tant qu'elle procède d'une ouverture au monde à l'œuvre dans la vie. Il ne s'agit naturellement pas, aux yeux de Jonas, de renoncer à "voir» mais plutôt de ne pas réduire le percevoir au seul voir et d'intégrer la vision dans un cadre perceptif plus large qui en destitue le monopole.

Sous cette condition, c'est en particulier l'expérience tactile qui se trouve revalorisée en ce qu'elle est l'occasion d'appréhender le mouvement même de la vie, par exemple dans l'expérience du choc, où une force se donne à sentir, et par laquelle se révèle de manière corrélative ma présence et celle du monde, mon engagement dans le

${ }_{18}$ PV, p. 154 et 157. 
monde et «l'effet » du monde sur moi. Ainsi si la vue est par excellence le sens qui rend possible notre rapport objectif à la nature ${ }^{19}$, c'est en revanche grâce au toucher que nous pouvons faire l'expérience des forces du monde ${ }^{20}$. Cette expérience de la force ne doit pas être interprétée en des termes mécanistes, puisqu'elle crée au contraire les conditions grâce auxquelles une objectivité pourra être construite $^{21}$. L'expérience que je fais du monde dans le toucher, qui suppose le mouvement corporel ${ }^{22}$, est la condition originaire à partir de laquelle la vision pourra constituer l'objectivité de la nature. C'est en effet sur la base de la mise en mouvement de mon corps touchant que peuvent être synthétisées, d'une part, les données de l'objet fournies par un seul et même sens et, d'autre part, les données des divers sens. Quand bien même la vue doive être considérée comme étant le plus objectivant de tous les sens, Jonas estime en effet qu'elle ne pourrait cependant exercer son office sans l'expérience d'un soi corporel se mouvant dans un espace que, par-là même, il ouvre et grâce auquel nous pouvons identifier des objets ${ }^{23}$. Jamais nous n'aurions par exemple le sentiment de la perspective, nécessaire à l'appréhension d'un objet, si nous n'avions, au moins, antérieurement, eu l'expérience du mouvement.

Pour autant, reconnaissons qu'une telle analyse, dont nous n'avons évoqué ici que les principes, suscite elle-même des réserves. En effet, il faut faire droit à une ambiguïté présente au sein même de la phénoménologie jonassienne, puisque tout en prétendant décrire l'ouverture au monde de la vie en prenant pour point de départ le témoignage de l'homme sur sa propre existence vitale, il continue de maintenir une conceptualité empruntée au langage objectivant, comme si donc la nature était déjà constituée lorsque la vie apparaissait en son sein et comme si le sujet devait s'expliquer à partir de ses lois. C'est ce qui apparaît clairement lorsque Jonas restitue l'explication scientifique en vertu de laquelle les grands organismes objectivent leur environnement du fait même de leur taille. Parce que chaque unité d'action présente dans l'activité perceptive voit son influence annulée au regard de la dimension de l'organisme, le vivant transforme une force en présence objective. Ainsi, alors que c'est la

\footnotetext{
${ }^{19} \mathrm{Cf}$. PV, Essai VI et appendice mais également l'Essai VII, p. 176-178.

${ }^{20} \mathrm{PV}$, p. 34 et 16 . Ce primat du toucher est également affirmé dans le manuscrit d'origine du Phénomène de la vie déposé aux Archives de Constance : HJ-10-16-2.

${ }^{21}$ PV, p. 162.

${ }^{22} C^{\prime}$ est en effet le mouvement volontaire de mon corps qui offre le cadre permettant d'unifier de manière cohérente la série de sensations tactiles. Cf. PV, p. 162.

${ }^{23}$ Cf. à ce propos PV, p. 162-165.
} 
force qui est première et l'entité persistante qui est résultat, voici que c'est au contraire l'entité qui paraît première, tandis que la force est expérimentée seulement dans les collisions ou les chocs ${ }^{24}$. C'est donc sur le terrain même de l'objectivité qu'il prétend paradoxalement décrire la genèse de l'objectivité.

On objectera certes que cette explication a pour fonction de désidéaliser l'interprétation idéaliste de la perception : la perception est le résultat de l'action d'un corps sur mon corps et non la conséquence d'un jugement intellectuel. Elle ne dispense pas et appelle même une description phénoménologique de l'activité perceptive, à même de décrire l'ouverture au monde dont notre corps est le lieu. Mais c'est précisément dans le cadre de cette description que la démarche de Jonas se révèle la plus ambiguë. Car tout en prétendant effectuer la genèse de l'objectivité à partir du mouvement vital de notre corps qui touche, tout en affirmant que c'est l'expérience tactile qui ouvre un espace et prépare l'objectivation dans laquelle la vision joue un rôle cardinal, tout en affirmant que la sensation devient perception par un effet d'abstraction qui présuppose originairement une expérience plus large de déploiement d'horizon, comme nous l'avons vu dans la description de l'activité métabolique, Jonas continue de décrire la transcendance de la vie vers le monde en termes de constitution objective. Tout se passe comme si, au moment même où il voulait rendre compte du fond sur lequel l'objet pouvait se détacher, il restait tributaire d'un langage qui fait de l'objet le terme même de la transcendance de la vie ${ }^{25}$. Très révélateur est à cet égard la reconnaissance implicite du découpage de l'activité perceptive entre les différentes activités sensorielles et de l'appréhension du champ perceptif à partir de champs sensibles préconstitués (le champ visuel, tactile). Au lieu de prendre pour point de départ le monde et de comprendre comment celui-ci constitue un champ originaire sur fond duquel les différents champs perceptifs sont possibles, Jonas entérine leur différence, considérée comme donnée et allant de soi, et tente de comprendre leur unité dans la visée synthétique d'objets. Les sens sont déjà posés dans leur séparation au sein d'un corps objet contenu dans une nature que ceux-ci sont censés atteindre en se donnant des objets.

Dès lors c'est ici la phénoménologie merleau-pontyenne qui retrouve tous ses droits en nous alertant sur la naïveté d'un tel

\footnotetext{
24 PV, p. 42 mais aussi p. 157.

${ }^{25}$ Ainsi le toucher qui est le sens par excellence faisant l'expérience infra-objective de la force est aussi le sens qui a accès à « la réalité " plutôt qu'à l'être (PV, p. 156) et le mouvement que nous expérimentons ouvre moins un monde qu'il ne permet la constitution de l'objectivité (PV, p. 162.).
} 
objectivisme. C'est que la perception ne doit pas être appréhendée comme appréhension d'un objet mais bel et bien comme expérience du monde. Ainsi le sensible doit être abordé à partir de la profondeur qui en préserve la transcendance, sous peine d'être immanentisé dans le vécu d'une conscience. Même si Jonas ne prétend pas partir de sensations toutes faites auxquelles se surajouteraient l'acte synthétique du jugement intellectuel afin d'expliquer l'apparition d'un objet $^{26}$, il conserve cette visée de l'objet et laisse dans l'impensé l'ouverture du champ originaire sur fond duquel une chose peut être perçue. C'est de manière purement nominale qu'il renvoie à la psychologie de la forme ${ }^{27}$, qui ne suffit d'ailleurs pas en elle-même à rendre compte du sens d'être de la forme, à partir de laquelle pourtant le sens du sentir est censé être décrit ${ }^{28}$. Les sensations sont donc posées et commence ensuite une activité de synthèse que prend en charge très essentiellement la faculté visuelle et plus originairement la faculté tactile, mais sans que soit explicité le fond originaire à partir duquel un champ tactile et un champ visuel peuvent se détacher, sans rendre compte du corps en tant qu'il peut donner lieu à l'ouverture de tels champs. Au contraire Jonas semble supposer la synthèse des sensations. C'est d'ailleurs ce qu'atteste le retour d'un vocabulaire de la "représentation» chez Jonas, lorsqu'il décrit l'image comme étant une copie de la « réalité » dite extérieure ${ }^{29}$.

$\mathrm{Au}$ contraire Merleau-Ponty se donnera pour tâche dans la deuxième partie de la Phénoménologie de la perception de rendre compte du sens d'être du champ phénoménal. La sensation ne doit pas être vue comme une qualité ou un état mais comme porteuse $\mathrm{d}^{\prime}$ une signification motrice ou vitale. Sentir une couleur, c'est donc $\mathrm{d}^{\prime}$ abord la vivre, mais non pas au sens husserlien d'une matière purement sentie que traverserait ensuite une intentionnalité lui donnant sens, la noèse faisant de la hulè un noème, le vécu atteignant la dignité du perçu. On voit mal en effet ce que serait une couleur vécue non perçue et pas davantage comment la transcendance de l'objet pourrait surgir de l'immanence du vécu ou se rajouter à elle. Vivre la couleur, c'est donc expérimenter un certain état de tension $\mathrm{du}$ corps. C'est d'emblée se porter par son corps vers un champ phénoménal autonome et non le comprendre à partir de la conscience. Il s'agit alors de retrouver la couche primordial du sentir

\footnotetext{
${ }^{26}$ PV, p. 187.

27 PV, p. 187.

${ }_{28}$ SC, p. 139-147.

${ }^{29} \mathrm{PV}$, Essai VII.
} 
avant même la division des sens, grâce à laquelle ceux-ci communiquent dans la perception de la chose. C'est le corps phénoménal qui progresse vers une synthèse, jamais achevée, mais qui a sa raison d'être dans la structure même de la chose visée. Les espaces sensoriels ne sont alors que les moments d'un même espace et leur différence fait sens sur fond d'un espace originaire dont l'unité est celle du monde lui-même. L'expérience du sentir est alors l'expérience d'une unité de tension du sujet corporel avec le monde et manifeste la transcendance d'un corps qui se porte vers le monde par ce moyen.

Alors que Jonas semble présupposer le sens d'être du monde en le réduisant à l'objectivité, Merleau-Ponty défait au contraire le caractère illusoire de cette objectivité en prenant pour thème la genèse même du monde, tel qu'il se donne au sujet incarné. Une telle remarque permet ainsi d'identifier le préjugé dont est victime Jonas à sa racine même. Certes, il convenait de comprendre le sujet en tant que vivant, afin d'échapper à la logique de mort qui reconduit l'opposition du sujet et de l'objet, mais en interprétant la vie à partir du métabolisme, interprétation éclairée par l'expérience que le sujet humain fait de sa propre vie, Jonas a tendance à compenser l'objectivisme légué par ce concept prélevé à la sphère biologique ${ }^{30}$ par une projection purement psychique, celle de la finalité que l'homme expérimente en lui-même, en tant qu'humain. Il renoue ainsi avec un dualisme implicite et comprend explicitement la vie comme finalisée pour avoir adopté un point de départ qui l'inclinait à un matérialisme qu'il fallait ensuite compenser. C'est ainsi que l'activité de renouvellement de «soi » que manifeste l'organisme est lue depuis l'expérience purement psychique de la cause finale.

Cette critique réciproque des tentatives de Merleau-Ponty et de Jonas dans leur prétention commune à penser une phénoménologie de la vie doit-elle se conclure sur une aporie ? Suggérons simplement pour conclure que le caractère inaccompli de ces tentatives pourrait résulter d'un oubli partagé. Pour décrire le phénomène de la vie à partir de lui-même, en échappant aux risques d'idéalisation et d'objectivation, il faudrait d'emblée reconnaître la dimension relationnelle du vivant, ce qui veut dire qu'une vie ne peut être appréhendée comme vie qu'à condition d'être saisie, dès l'origine, dans sa relation constitutive aux autres vivants. Ce ne serait pas du monde

\footnotetext{
${ }^{30}$ Jonas reconnaît que le matérialisme est un point de départ plus légitime que l'idéalisme (PV, p. 30). Mais on peut alors anticiper que sa critique du matérialisme le conduit à rester dépendant de certains de ses présupposés.
} 
que naît le sujet ni du sujet que naît le monde mais ce serait à partir de la relation qui existe entre les "sujets ", en tant que vivants, qu'il nous serait possible de parler de monde. La vie serait précisément cette ouverture au monde qui provient de la relation des sujets entre eux $^{31}$.

31 Cet article a été élaboré dans le cadre du projet de recherche no 3130574 du ConicytFondecyt. 